\title{
Complete System for Wireless Powering and Remote Control of Electrostatic Actuators by Inductive Coupling
}

\author{
Philippe Basset, Andreas Kaiser, Member, IEEE, Bernard Legrand, Dominique Collard, Member, IEEE, \\ and Lionel Buchaillot
}

\begin{abstract}
This paper proposes a successful asynchronous remote powering and control of electrostatic microactuators, organized in two distributed micro motion systems (DMMS) with the aim of realizing a wireless microrobot. Remote powering of the integrated circuit (IC) and the microelectromechanical systems (MEMS) components is obtained by inductive coupling at $13.56 \mathrm{MHz}$, and the digital transmission is created by modulating the carrier amplitude by $25 \%$. The system includes a high-voltage controller IC. It provides a link between the power and data on the receiver antenna on one side, and the actuators of the microrobot on the other. The micromachined antenna is designed to optimize the inductive coupling. The main IC building blocks, such as the received signal rectifier/amplifier, the integrated digital processing and the DMMS actuation voltage generation are given in detail. The demonstrator has successfully achieved the remote control and asynchronous operation under $100 \mathrm{~V}$ of two arrays of 1700 electrostatic actuators, having a capacity of $2 \mathrm{nF}$ each.
\end{abstract}

Index Terms-Distributed micro motion system (DMMS), electrostatic actuator, high-voltage IC, microelectromechanical system (MEMS), microrobot, remote control, wireless powering.

\section{INTRODUCTION}

$\mathbf{T}$ HE use of electromagnetic coupling properties in microelectronics has considerably increased in the last ten years because it not only permits supply of electronic circuits remotely, but also exchange data between two devices. Today in the industry, the most important part of inductive coupling appli-

Manuscript received October 24, 2005; revised April 29, 2006. Recommended by Technical Editor C. Cecati. This work was supported by the Centre National pour la Recherche Scientifique (CNRS).

P. Basset was with the Institut d'Electronique, de Microélectronique et de Nanotechnogie, Unité Mixte de Recherche Centre National de la Recherche Scientifique (IEMN UMR CNRS), 59652 Villeneuve d'Ascq Cedex, France. He is now with the ESYCOM Laboratory, Ecole Supérieure d'Ingénieurs en Electronique et Electrotechnique (ESIEE), 93162 Noisy-le-Grand Cedex, France (e-mail: p.basset@esiee.fr).

A. Kaiser is with the Institut d'Electronique, de Microélectronique et de Nanotechnogie, Unité Mixte de Recherche Centre National de la Recherche Scientifique (IEMN UMR CNRS), 59652 Villeneuve d'Ascq Cedex, France, and also with the Institut Supérieur d'Electronique du Nord (ISEN), 59046 Lille Cedex, France.

B. Legrand and L. Buchaillot are with the Institut d'Electronique, de Microélectronique et de Nanotechnogie, Unité Mixte de Recherche Centre National de la Recherche Scientifique (IEMN UMR CNRS), 59652 Villeneuve d'Ascq Cedex, France.

D. Collard is with the Institut d'Electronique, de Microélectronique et de Nanotechnogie, Unité Mixte de Recherche Centre National de la Recherche Scientifique (IEMN UMR CNRS), 59652 Villeneuve d'Ascq Cedex, France, and also with the Center for International Research on Micromechatronics, Institute of Industrial Sciences, University of Tokyo, Tokyo 153 8505, Japan.

Digital Object Identifier 10.1109/TMECH.2006.886245 cations is related to radio frequency identification (RFID) [1]. RFID is used for contactless smartcards or to sort and locate traveling objects, e.g., like postal parcels. In many buildings, door keys have been replaced by an electromagnetic badge, and it is envisaged that magnetic RFID tags will replace bar codes soon. Power transmission and remote control by inductive coupling are also widely used in the field of sensors [2]. Recently, the car industry has realized a system, controlling the tire pressure of a vehicle in real time by means of an inductive link between a sensor and the car computer. Many possibilities are foreseen in medical applications, such as permanent implants, supervising various parameters like the insulin rate for diabetic or the intraocular blood pressure [3]-[5]. Other reported studies are about the recording of the neuronal activity [6] or the excitation of a retina with defective photoreceptors [7]. However, the remote powering and simultaneous control of micromechanical actuators still demands attention. Numerous applications can be envisaged if remote control is provided to autonomous microrobotic systems, notably in the fields of microassembly and testing of equipments, and circuits in confined environments [8].

Several microrobots have been fabricated over the past ten years, using various actuation means like mechanical transduction [9], thermal expansion [10], [11], or electrostatic forces [12], [13]. The major difficulty in remotely powering these devices is still the amount of power that needs to be transmitted: according to the type of actuation, it can vary from a few hundred milliwatts to several watts [14], [15]. If the majority of inductive links relates to low-power applications, by optimizing the size and the quality factor of the receiver antennas, as well as the emitter power, several watts can be transmitted over several centimeters [16]. Successful remote powered motion by inductive link has been demonstrated for impact drive [12] and electrostatic comb drive [17] actuators. In 2003, Hollar et al. presented the first powered autonomous silicon microrobot, thanks to photocells [13]. In those three examples, only a very limited number of actuators were simultaneously powered and no asynchronous control was performed.

Like in microelectronics, with the integration on a silicon substrate and mass production, new concepts are developed by multiplying the number of actuators carrying out complex actions, or assembling them in series or in parallel to obtain a macroscopic effect [18]. The need to correctly coordinate the actuators and to establish communication between the sensors/actuators and a remote controller requires "intelligence" in the system. Thus 
arises the idea of distributed and autonomous micromachines [autonomous distributed microsystems (ADMs)]. An ADM is a system made up of several intelligent subsystems, which can share information and cooperate. As a first step toward ADMs, Fujita et al. [19]-[22] developed the concept of distributed micromotion system (DMMS). The governing idea is to coordinate a significant number of small actions (in this case, displacements), in order to obtain a more complex function. As demonstrators, several microconveyance systems were built using the DMMS concept. They used either thermal actuators reproducing the ciliary movement occurring in living organisms [14], or an air flow controlled by multiple microvalves [20], [22] as well as electrostatic [23], [24] and magnetic [25] actuation. In such a system, the distributed actuators are fixed, and appropriate control is provided to move a micro object. A further step toward ADMs is the attempt to build microrobots with remote power supply and remote control. In that case, additional difficulties arise from asynchronous command requirements, as it will be mandatory to actuate microelectromechanical system (MEMS) devices with coordinated and independent actions in order to carry out tasks with increased complexity. A technique using a single-emitter frequency per actuator was proposed [17], which requires, in the case of multiple simultaneous commands, a complex set of several emitter/receiver systems.

Only few remote powering experiments of individual microactuators have been realized so far [12], [13], [17], and no complete protocol of communication for both powering and controlling multiple independent actuators have been reported yet. To more deeply investigate this topic and to make possible the operation of autonomous microrobots incorporating DMMS capabilities, this paper proposes and demonstrates a complete approach based on magnetic coupling for both powering and asynchronous control of integrated microactuator arrays. This paper has been driven by the concept of a mobile autonomous microrobot that is described in Section II. Section III details the fabrication process of two distributed arrays of electrostatic actuators. They will be used to validate the system. Section IV deals with the remote powering and wireless data transfer issues. Section V describes a high-voltage (HV) integrated circuit (IC) dedicated to the remote control of electrostatic actuators and Section VI describes the results of the successful asynchronous actuation of the two DMMS.

\section{Autonomous Robot Concept}

The autonomous robot concept for which the system has been developed is depicted in Fig. 1. By means of an antenna, the microrobot captures part of the electromagnetic energy to provide power for its own operation. One of the advantages of this approach is the ability to establish bidirectional communication between the microrobot and the remote controller. Amplitude modulation of the magnetic field is used for the downlink (controller to microrobot), while a simple load modulation at the microrobot antenna provides the uplink (microrobot to controller). The power consumption for the communication on the robot side is very small, as it is not required to radiate power. This concept

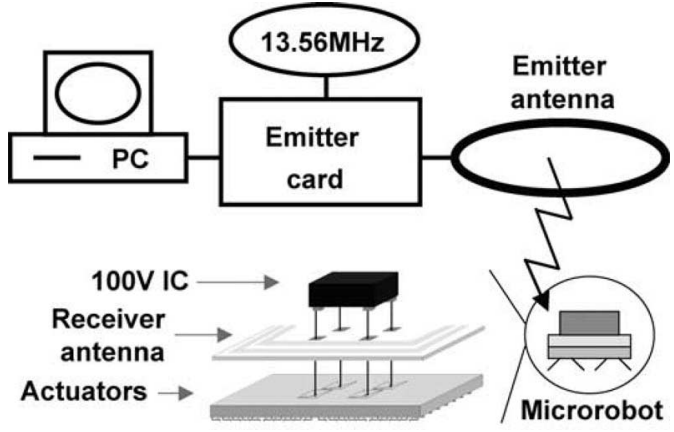

Fig. 1. Overview of the autonomous microrobot concept.

does not basically allow direct communication between several microrobots present in the same electromagnetic field. However, system level protocols, such as those in use in contactless smart-card readers, allows a single controller to communicate with several individually identified microrobots. These protocols could be exploited for the construction of an ADM, where a number of intelligent microrobots can cooperate using the controller as a communication relay. Whatever may be the motion system of the microrobot, an asynchronous command is needed to obtain a two-degrees-of-freedom displacement. The complete system, described in this paper, allows the remote actuation of four actuators with actuation voltages of up to 100 $\mathrm{V}$. The commands are sent from the PC to the actuators in the form of an amplitude-modulated signal. The maximum transmit power is $25 \mathrm{~W}$. The mobile part has a micromachined receiver antenna made of gold and patterned on an insulate substrate of epoxy. This antenna is assembled with a HV IC and the DMMS array.

\section{DMMS}

In order to test the asynchronous remote control of complex microrobotic systems, two arrays of 1700 electrostatic actuators, covering a surface of $32 \mathrm{~mm}^{2}$, have been fabricated. They correspond to the motion system of the microrobot. These DMMS are made of modified inverted scratch drive actuators (SDA) [24], [26]. This section presents the principle of the actuator and its fabrication process.

\section{A. Description of the Actuator}

The actuator is composed of a clamped-free plate partially covering a buried electrode, as shown in Fig. 2. The applied voltage between the plate and the electrode enables the pullin of the moving part toward the substrate due to electrostatic forces. When the stopper contacts the substrate, an axis of torsion is created, which induces the rise of the plate's free end, with $x$ and $z$ displacements. An elevator is added to compensate for the gap between the stopper and the substrate. This actuator performs adequate movement for a ciliary motion system (CMS) [14] 


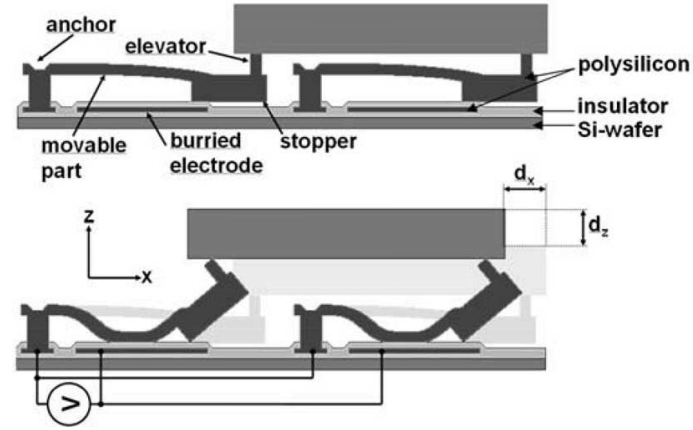

Fig. 2. Actuator principle.

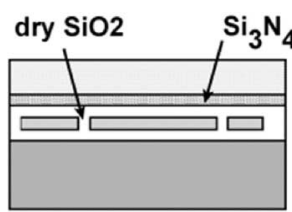

(a)

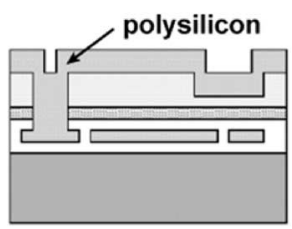

(c)

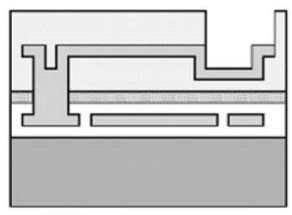

(e) (b)

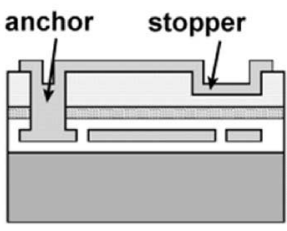

(d)



(f)

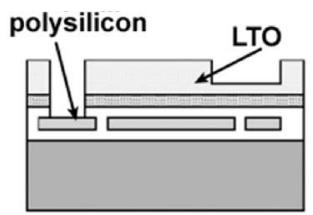

Fig. 3. Process steps.

\section{B. Fabrication Process}

The actuator is built from a standard polysilicon/oxide surface micromachining process [27] with an additional level of polysilicon for the elevator. Six photolithography masking steps are needed. A cross section of the actuator at the main steps in the process sequence is given in Fig. 3. On a silicon substrate, first a layer of dry $\mathrm{SiO}_{2}$ is grown. A thin layer of doped polysilicon is deposited by low pressure chemical vapor deposition (LPCVD), and is patterned by reactive ion etching (RIE) to create the buried electrodes. These electrodes are covered with dry oxide and nitride bilayers. A 1.5- $\mu \mathrm{m}$-thick layer of low temperature oxide (LTO) is then deposited, which serves as a sacrificial layer [Fig. 3(a)]. This oxide is etched in two steps to obtain the mold of the stoppers and to form the anchors with the buried electrodes [Fig. 3(b)]. Then, a 1.5- $\mu$ m-thick structural LPCVD polysilicon is deposited, doped by phosphorus implantation and annealed at $1000{ }^{\circ} \mathrm{C}$ for $60 \mathrm{~min}$ for internal mechanical stress relaxation [Fig. 3(c)]. The structural layer is patterned by RIE and the polysilicon thickness is then reduced from 1.5 to $1 \mu \mathrm{m}$ by isotropic $\mathrm{SF}_{6}$ plasma etching [Fig. 3(d)]. This thinning aims at reducing the pull-in voltage of the actuators [27]. A second 1.5- $\mu \mathrm{m}$ LTO layer is deposited, and then, etched by RIE to form the elevator molds [Fig. 3(e)]. Short dips in $\mathrm{HNO}_{3} / \mathrm{HS}_{2}$ and $\mathrm{HF}$ baths make sure that no residual resist or oxide remains at the bottom of the mold before performing the last polysilicon deposition. Then, the elevators are patterned and the two sacrificial oxide layers are dissolved in HF [Fig. 3(f)].

\section{Experimental Results}

Arrays of $1-\mu \mathrm{m}$-thick actuators with a length of $125 \mu \mathrm{m}$ and a stopper height of $1.5 \mu \mathrm{m}$ have been fabricated. An actuator width of $60 \mu \mathrm{m}$ has been chosen for easy release of the structure. Actuations of the DMMS have shown a pull-in voltage of $50 \mathrm{~V}$. When loaded with a $380-\mu \mathrm{m}$-thick silicon piece of $0.25 \mathrm{~mm}^{2}$, the pull-in voltage becomes $80 \mathrm{~V}$.

\section{REMOte POWERING AND DATA TRANSFER}

Remote powering of the IC and the MEMS components is obtained by inductive coupling at $13.56 \mathrm{MHz}$ (generic frequency for RFID applications in the megahertz range). Digital data is transmitted by modulating the carrier's amplitude by $25 \%$, so that there is always power provided to the chip. Fig. 4 shows the block diagram of the emitter circuit. The modulating signal is a serial-bit sequence containing the data (on or off) for the actuation of the four MEMS devices, and is sent continuously.

\section{A. Emitter Board}

Initially, the microcontroller acquires the actuation voltage, the modulation index, the modulation frequency and the command data from the PC interface. It then generates an 8-b control stream that directly modulates the amplitude of the $13.56-\mathrm{MHz}$ carrier. The modulated signal is amplified and, lastly, an output buffer provides a signal of up to $1 \mathrm{~A}$ to the emitter antenna. The command data, modulation index, and data rate can be modified at any time on the PC.

The emitter antenna, made of four 7-cm-diameter loops of a 1-mm-thick copper wire, must be perfectly adapted to the output impedance of the circuit to optimize the power transfer. Impedance matching is obtained using the RLC network of Fig. 5, derived from the T-match circuit [28]. $Z_{i n}$ is purely real when the reactive components are perfectly symmetrical. The capacitor $C_{1}$ is needed to allow independent adjustment of impedance, signal frequency, and antenna inductance. Assuming its quality factor $Q$ is higher than ten, component values are easily calculated using serial/parallel transformations of RLC networks.

\section{B. Design and Fabrication of the Receiving Antenna}

The receiving antenna is a key element of the system, as the power transmission is highly dependent on its $Q$. The electrical model of the inductive link, (Fig. 6) is composed of a loop antenna $L_{1}$ driven by a sinusoidal current $i_{1}$ of radian frequency $\omega$, and a second inductive loop antenna $L_{2}$ realizing a RLC tank with a parallel tuning capacitor $C_{2}$ and a load resistance $R_{L}$. 


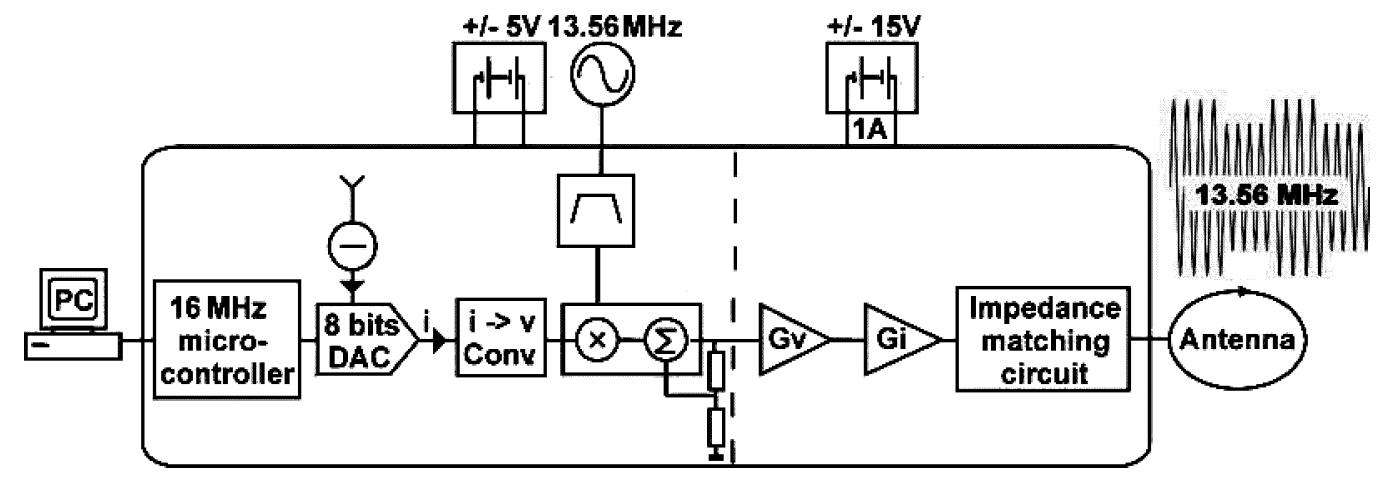

Fig. 4. Block diagram of the emitter board.

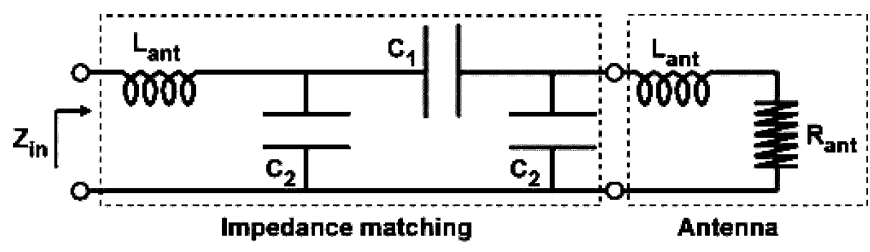

Fig. 5. Impedance matching circuit of the emitter antenna.

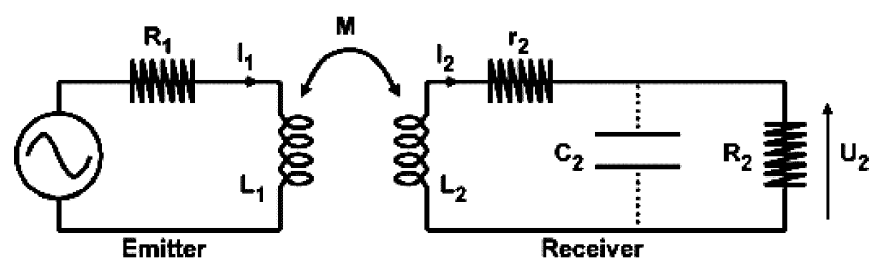

Fig. 6. Electrical model of the inductive link.

According to [29], the induced voltage $u_{2}$ across $L_{2}$ is given by

$$
u_{2}=\frac{j \omega k \sqrt{L_{1} L_{2}}}{1+\frac{r_{2}}{R_{L}}-\omega^{2} C_{2} L_{2}+j \omega\left(C_{2} r_{2}+\frac{L_{2}}{R_{L}}\right)} i_{1}
$$

where $r_{2}$ is the serial loss of $L_{2}$ and $k$ is the coupling factor between the two loops. If the resonant frequency of the tank is tuned to be equal to $\omega$, the maximal value of $u_{2}$ becomes

$$
\left|u_{2 \max }\right|=\frac{\omega_{r} k \sqrt{L_{1} L_{2}}}{\sqrt{\left(\frac{r_{2}}{R_{L}}\right)^{2}+\omega_{r}^{2}\left(C_{2} r_{2}+\frac{L_{2}}{R_{L}}\right)^{2}}} i_{1}
$$

where $\omega_{r}=\left(L_{2} C_{2}\right)^{-1 / 2}$. This expression of the induced voltage shows the main issues when designing an antenna for inductive coupling: in addition to maximizing the coupling factor, it is critical to minimize the serial resistive loss of $L_{2}$ and to maximize the ratio $L_{2} / C_{2}$ for a given resonance frequency, particularly if $R_{L}$ is large.

To be easily integrated with MEMS actuators, the receiver antenna has been fabricated using classical microtechnologies. For a given area, antenna design requires tradeoff between many parameters such as the number of turns, the conductor width, and the gap between two adjacent turns. The maximization of the $Q$-factor of a planar coil requires both a small gap between two turns in order to have a strong coupling of the magnetic field, and a wide conductor to reduce the ohmic losses. However, Craninckx and Steyaert [30] showed that the resistive serial losses increase with frequency, and this increase is enhanced in the case of a coil made of a wide conductor. Moreover, very close spacing of turns will increase the interturn parasitic capacitance. Also for a given area, wider conductors lead to a reduced number of turns, thus, the inductance will decrease while, the parasitic substrate capacitance will increase. It is actually convenient to remove the interior turns of the antenna in order to increase the $Q$-factor, as these turns contribute only marginally to the total inductance.

Due to the discussed design tradeoff, the receiving antenna is a square coil where the inner turns are removed. It is made of electroplated gold and fabricated on an insulating substrate to prevent Eddy currents. First, a $2-\mu \mathrm{m}$ LTO is deposited on a silicon wafer and subsequently used as a sacrificial layer. Then, $150 \mu \mathrm{m}$ of epoxy-type resist SU-8 is spun up. This film, once polymerized, becomes a flexible, tough, and light substrate. The conductor is made of $24-\mu \mathrm{m}$-thick electroplated gold. To achieve the electrolysis, a seed layer of nickel is sputtered. A 30- $\mu \mathrm{m}$ thick mold of positive resist AZ4562 is created and followed by gold plating. Finally, the mold is removed, the seed layer of nickel is etched by nitric acid, and the SU-8 film is separated from the silicon using hydrofluoric acid (HF). The details of the process can be found in [31].

\section{HV CONTROLLER IC}

The controller IC is the heart of the remote subsystem. It provides the link between the power and communications available at the receiving antenna on one side, and the actuators or possibly sensors at the other side. It also provides the local intelligence of the system. The circuit block diagram is given in Fig. 7, and a detailed microphotograph is displayed in Fig. 8. The circuit integrates the following functions: 1) amplification, rectification, and limitation of the induced voltage to supply the power; 2) clock recovery from the carrier and $5-\mathrm{V}$ regulation for the digital part; and 3) demodulation of the received signal, digital processing, and driving of the actuators. In the case of sensors added to the system, an antenna load modulation circuit can easily be added to provide a communication link from the sensors to the emitter station. The circuit implementation, dedicated to micromechanical electrostatic actuation, has been optimized to 


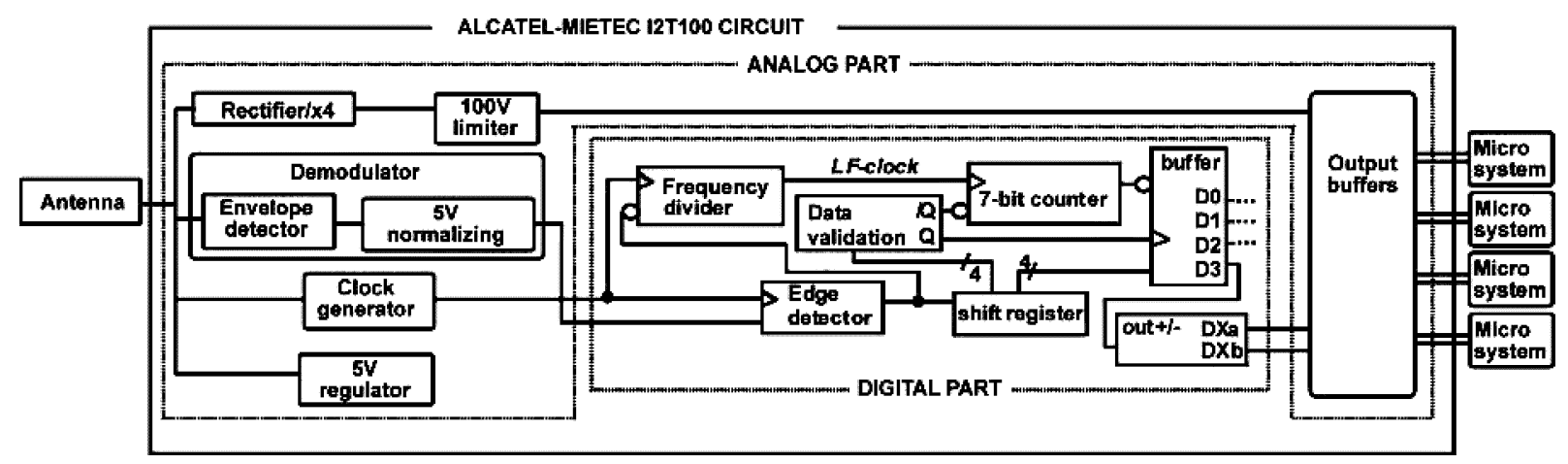

Fig. 7. Block diagram of the $100-\mathrm{V}$ telemetry IC.

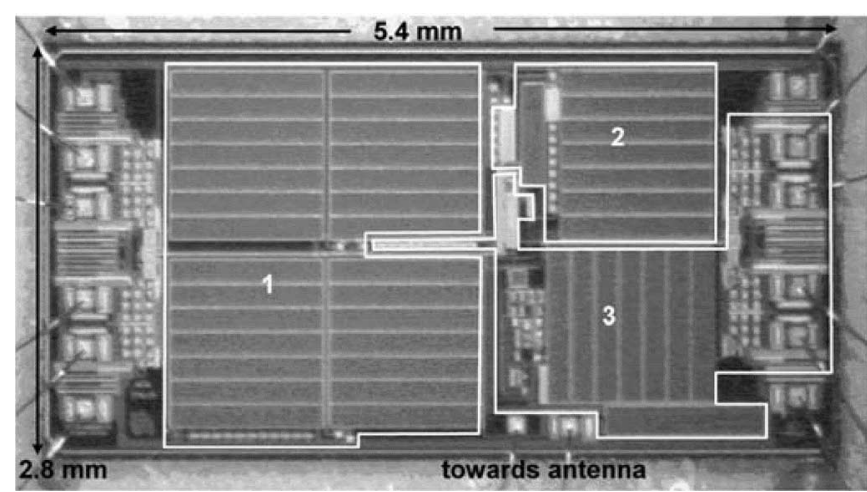

Fig. 8. Microphotograph of the IC. 1-rectifier and limiter of the induced voltage, 2 - clock recovery and 5-V regulator, and 3-demodulator, digital processing, and HV output buffers.

drive "charge controlled" devices, requiring no dc power and allowing high-actuation voltage outputs (typically $>30 \mathrm{~V}$ ) with a symmetrical signal to avoid charging effects in the electrostatic actuator. The upper limit of the driving voltage is fixed by the fabrication technology. Here, the circuit was fabricated in the I2T100 process of AMI semiconductors. This process combines $0.7-\mu \mathrm{m}$ CMOS, medium-voltage bipolar transistors, and complementary lateral double-diffused metal-oxide-semiconductor (LDMOS) high-voltage transistors with a maximum allowable drain-source voltage of $100 \mathrm{~V}$, which is the maximum available driving voltage.

\section{A. Analog Circuit}

The simplified schematics of the different analog circuits are given in Fig. 9. A charge-pump system made of two CockroftWalton rectifiers are implemented in series in order to provide a dc voltage that ideally is four times the peak voltage at the receiving antenna inputs [see Fig. 9(a)]. However, as we will see in Section VI, parasitic capacitances of the HV diodes will drastically limit that gain. In order to drive highly capacitive microsystems, $C_{1}-C_{4}$ have high values and occupy most of the chip's area, as shown in Fig. 8. The Zener diodes limit the output voltage to $100 \mathrm{~V}$. A 5-V regulator [Fig. 9(c)] and a clock generator at the carrier frequency [Fig. 9(d)] allow the operation of the digital block.

The demodulator [Fig. 9(b)] recovers the carrier's envelope with a diode followed by a low-pass filter, and transforms the amplitude modulation to a $0 / 5-\mathrm{V}$ signal for subsequent digital processing. The demodulation compares the envelope with a voltage ranging between the high and low levels of the signal. This intermediate voltage cannot be averaged with an $R C$ time constant. This would demand a too large a chip area, and would fix the modulation index. A substitutive circuit is composed of two diodes in parallel with opposite polarity between the $(+)$ and the $(-)$ inputs of the comparator, in series with a capacitor between the (-) entrance and the ground. Hence, for a high-level state transmission, the voltage $V_{c}$ across the capacitor is $\left(V_{\mathrm{env}}-V_{z}\right)-V_{t}$, so $V_{d}=V_{t}$, and $V_{\mathrm{env}}, V_{t}, V_{z}$, and $V_{d}$ are, respectively, the envelop voltage, the threshold voltage of the diode-connected MOS transistors, the breakdown threshold of the Zener diode, and the voltage difference between the inverting and noninverting inputs of the comparator. When the following low-level state occurs, if the modulation index is high enough, $V_{\text {env }}-V_{z}$ becomes lower than $V_{c}$, which moves to $\left(V_{\text {env }}-\right.$ $\left.V_{z}\right)+V_{t}$ and $V_{d}=-V_{t}$. The data are then normalized to a 0/5-V square signal for the digital block. The HV buffer [Fig. 9(e)] transforms the command coming from the digital part into a voltage equal to the rectifier output voltage. This interface is similar to a CMOS inverter, except for its gate control, which needs two signals, one for the N-DMOS of the inverter that is directly controlled by the digital block, and one for the P-DMOS that needs a level shifter since its source is connected to the HV. Details of this circuit can be found in [32].

\section{B. Digital Circuit}

A 10-b frequency divider converts the $13.56-\mathrm{MHz}$ clock to $13.2 \mathrm{kHz}$, the low-frequency (LF) clock. A reset of the divider occurs at each edge detection of the signal coming from the demodulator to constantly synchronize the data with the clock. The serial/parallel conversion of the data is carried out through eight $D$-flipflops in series, which are synchronized with the LF clock. They implement an 8-b shift register. Four bits of data $D_{0}-D_{3}$ are needed here to command four independent groups of actuators. These bits are sent together at the synchronization frequency, encapsulated with four other data validation markers to form the byte " $10 D_{3} D_{2} 0 D_{1} D_{0} 1$." This byte is continuously entering into the register. At each LF-clock period, the location in the register of the additional bits is checked. If it is recognized as being correct, the data is sent to the output buffer. 


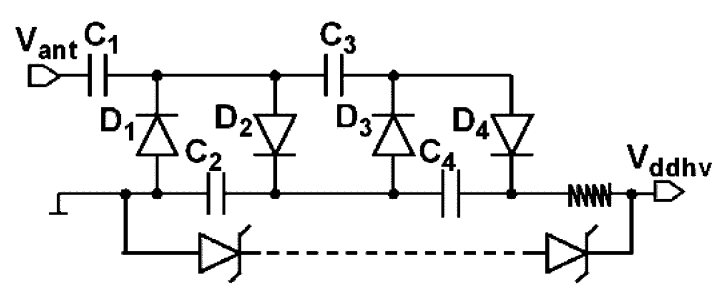

(a)

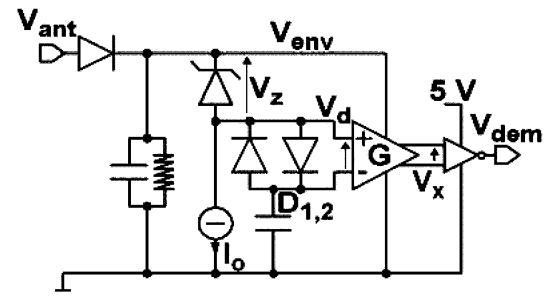

(b)

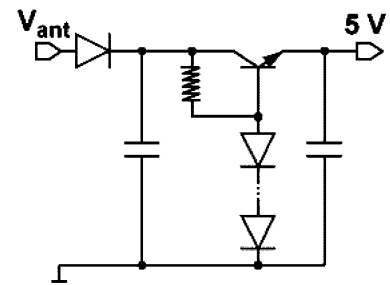

(c)

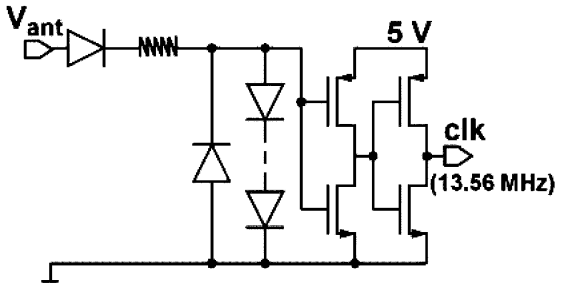

(d)

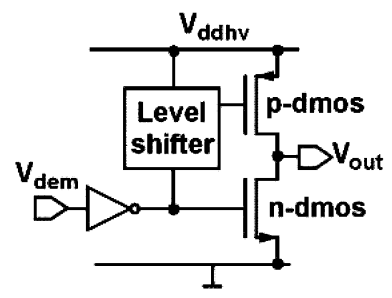

(e)

Fig. 9. Simplified schematics of the analog circuits. (a) Multiplier/rectifier. (b) Demodulator. (c) 5-V regulator. (d) Clock generator. (e) HV output buffer.

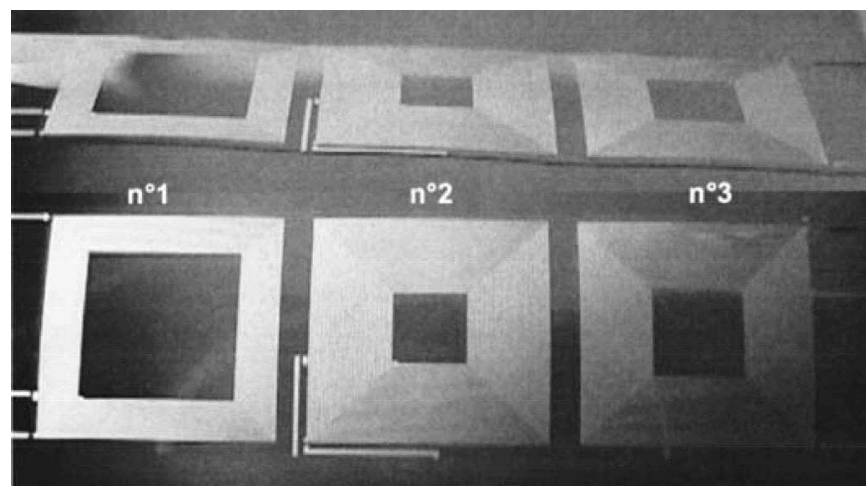

Fig. 10. Photograph of the antennas.

TABLE I

Characteristics OF THE RECEIVER ANTENNAS

\begin{tabular}{lcccc}
\hline \hline & Antenna 1 & Antenna 2 & Antenna 3 \\
\hline Coil dimension $\left(\mathrm{mm}^{2}\right)$ & $15 \times 15$ & $15 \times 15$ & $15 \times 15$ \\
Number of turns & 16 & 26 & 23 \\
Width of gold & 62.5 & 100 & 100 \\
traces $(\mu \mathrm{m})$ & & & \\
Gap between & 100 & 100 & 100 \\
traces $(\mu \mathrm{m})$ & 5.8 & 7.8 & 7.2 \\
$\mathrm{~L}(\mu \mathrm{H})$ & 54 & 49 & 62 \\
Self Resonance & 1.5 & 1.3 & 0.92 \\
Frequency $(\mathrm{MHz})$ & 19 & 27 & 29 \\
$\mathrm{C}(\mathrm{pF})$ & 27 & 32 & 37 \\
$\mathrm{Q}_{\mathrm{L}}(13.56 \mathrm{MHz})$ & $(27 \mathrm{MHz})$ & $(15.5 \mathrm{MHz})$ & $(19 \mathrm{MHz})$ \\
$\mathrm{Q}_{\mathrm{L}}$ max & \multicolumn{3}{|c}{} \\
\hline \hline
\end{tabular}

During the charging of a capacitive load, the received voltage could drop due to the high current consumption. To avoid misinterpretation of the input code, the data transmission from the analog to the digital circuit is disabled for that time.

Electrostatic stiction or hysteresis are unfortunately common in MEMS devices, especially with polysilicon-based electrostatic actuators. This drawback is due to charge accumulation
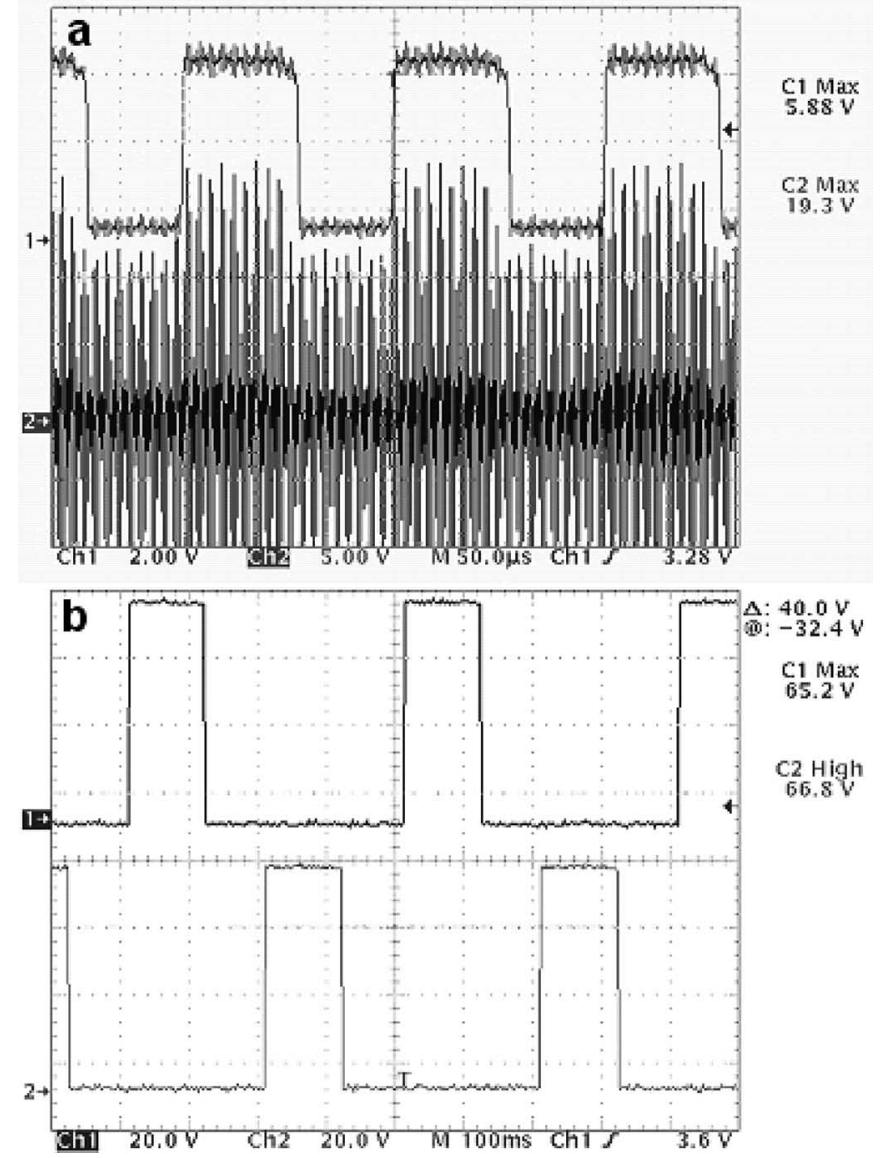

Fig. 11. Oscillograms of the HV IC. (a) Input voltage and demodulator output. (b) Two outputs corresponding to the two electrodes of one device (antistiction actuation) for a voltage gain of 3.5 .

in the dielectric, and it can be limited by alternating the polarity of the command on each electrode [33]. This function is realized by the out $+/-$ block of Fig. 7. For successive rising edges on a same data input $D_{X}$, the actuation voltage is applied 




Fig. 12. Photograph of the experimental setup.

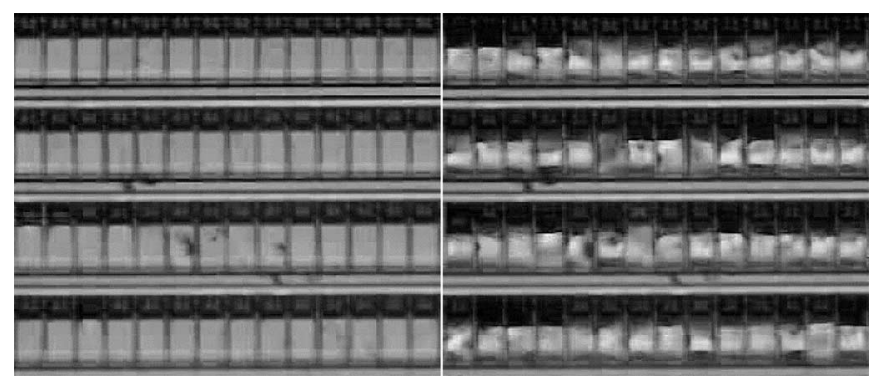

Fig. 13. Video shots of the remote actuation of one DMMS.

alternatively on the outputs $D_{X a}$ and $D_{X b}$. During a transmission without modulation, the register, which contains the last valid byte, can stay unchanged for a long time. A second antistiction circuit acts to reset the buffer after 128 unsuccessful byte receptions. The actuators are then relaxed, if no data is transmitted.

\section{EXPERIMENTS}

\section{A. Test of the Antennas}

Different designs of hollow coils have been fabricated and released from the silicon substrate. Fig. 10 shows three antennas with an outer diameter $d_{0}$ of $15 \mathrm{~mm}$ and a gap between the gold turns of $100 \mu \mathrm{m}$. The turn number of antennas referenced 1,2, and 3 are, respectively, 16, 26, and 23, and the conductor widths are $62.5,100$, and $100 \mu \mathrm{m}$. Antenna testing was performed with an Agilent 8753ES network analyzer. Oneport $S$ parameters were measured from $30 \mathrm{KHz}$ to $100 \mathrm{MHz}$ and converted to impedance $Z_{L}$. Then, the parasitic capacitance of the test device was de-embedded using open calibration of the device. Parameter extraction is deduced from an equivalent circuit consisting of a capacitor $C$, in parallel with an inductance $L$ and in series with a resistor $R$. As frequency increases, the penetration of the magnetic field into the conductor is attenuated (skin effect), which causes a reduction in the magnetic flux internal to the conductor. However, $L$ does not decrease significantly with increasing frequency because it is predominantly determined by the magnetic flux external to the conductor [34]. Thus, $L$ can be approximated as remaining constant with frequency. $C$ is considered independent of the frequency since it represents the metal-to-metal overlap capacitance between the turns. $L$ is extracted from the LF imaginary part of $Z_{L}$, and $C$ is extracted using the LF $L$ value and the resonance frequency of the antenna. The quality factor $Q_{L}$ is estimated by taking the ratio of the imaginary and real parts of the one-port impedance, observing that $Z_{L}$ is purely inductive in the frequency range of interest. These results are summarized in Table I.

\section{B. Test of the IC and Remote Asynchronous Operation of Multiple Electrostatic MEMS}

Two large DMMS, made of 1700 actuators and covering a surface of $32 \mathrm{~mm}^{2}$, have been fabricated to demonstrate the asynchronous operation of MEMS devices via inductive coupling using the dedicated IC. They have been alternately remotely actuated at a distance of $1 \mathrm{~cm}$. For a pure capacitive load, the minimum modulation factor is $6 \%$, which results from the threshold voltage of the diodes $D_{1}$ and $D_{2}$, as shown in Fig. 9(b). The optimum index is obtained for a $25 \%$ modulation. This index is defined as the minimum modulation that guaranties both correct demodulation and maximum received voltage on the antenna. When the load is resistive, the input voltage decreases due to the quality factor degradation of the input RLC tank. If the low-level input voltage decreases below $11 \mathrm{~V}$, the modulation rate has to be increased. The demodulator works correctly as long as the high-input level is ranging between 11 and $20 \mathrm{~V}$, as shown by the oscillograms in Fig. 11(a). The 5-V regulator generates a voltage ranging between 4.96 and $5.44 \mathrm{~V}$. Due to underestimated parasitic capacitances of HV diodes and capacitors with the substrate, the rectifier presents a linear gain of 1.4 for a predicted value of four, and the clock is filtered above a few tens of kilohertz. However, a gain of three, using integrated diodes, could easily be implemented by optimizing the polarity of the HV capacitors in order to avoid capacitive dividers. The digital part works for a modulation frequency precisely equal to $1 / 1024$ times the frequency of the carrier. The IC was successfully tested with capacitive loads up to $220 \mathrm{nF}$ without any effect like collapsing of the received voltage due to the output current. The rectifier input capacitance and output resistance are $88 \mathrm{pF}$ and $18 \mathrm{k} \Omega$ espectively. To compensate for the increased parasitic input capacitance of the rectifier, the integrated antenna has been replaced by a $2 \times 2 \mathrm{~cm}^{2}$ hollow coil of $1.6 \mu \mathrm{H}$. A maximum actuation power of $23 \mathrm{~mW}$ has then been obtained with highly resistive load $(>100 \mathrm{k} \Omega$ ). Due to low gain of the integrated rectifier, an external multiplier made of discrete components has been added to test the HV output actuation. Fig. 11(b) represents the oscillogram of two outputs corresponding to the two electrodes of one actuator 
array (antistiction actuation) for a total voltage gain of 3.5. The voltage is alternatively applied on the two electrodes of each DMMS, and electrostatic hysteresis or stiction of the mobile parts have been successfully avoided. The capacitance of the actuator array, calculated close to $2 \mathrm{nF}$, generates a large $R C$ constant. For that reason, the DMMS can not be actuated above $20 \mathrm{~Hz}$. Photographs of the test experiment are shown in Fig. 12, and Fig. 13 shows two video shots of the DMMS, one during the relaxation time and the other during the actuation.

\section{CONCLUSION}

A complete system for wireless powering and remote control of electrostatic actuators using radio-frequency waves has been presented in this paper. The underlying principles are similar to contactless smartcards. However, the required power levels are higher due to the need to drive actuators. The key elements enabling the remote powering are a high-performance integrated antenna and an appropriately integrated mixed-signal controller circuit, able to convert the received energy to the required voltage levels. The antenna is made of thick electroplated gold on an epoxy substrate to enhance its $Q$-factor by preventing Eddy currents. The dedicated IC allows asynchronous actuation of four remote devices with specific features for electrostatic MEMS, such as voltages up to $100 \mathrm{~V}$, antistiction functionalities, and the ability to drive highly capacitive loads. For the purpose of demonstration, two DMMS of 1700 electrostatic MEMS have been alternatively actuated at a distance of $1 \mathrm{~cm}$, thanks to a $2 \times 2 \mathrm{~cm}^{2}$ hollow coil antenna and a $9-\mathrm{W}$-powered emitter board. The power efficiency remains quite poor, which is related to the small size of the system, and the consequently small size of the antenna. The large capacitance of the tested DMMS induces a maximum operating frequency of $20 \mathrm{~Hz}$. According to the finite element method (FEM) results of the actuator's $x$ displacement [26], such a DMMS-based microrobot supporting a load of $2.5 \mu \mathrm{N}$ is expected to move at a speed of $4.8 / 3 \mu \mathrm{m} \cdot \mathrm{s}^{-1}$ for a 90/125 actuator length, respectively.

The possibility to establish communication channels between multiple devices in the same electromagnetic field opens new perspectives, namely, the collective operation and collaboration of sensors for smart dusts [35] or micromotion system.

\section{ACKNOWLEDGMENT}

The authors would like to thank S. Arscott, P. Bigotte, and the whole technical staff of IEMN for their contribution to the achievement of the process.

\section{REFERENCES}

[1] U. Kaiser and W. Steinhagen, "A low-power transponder IC for highperformance identification systems," J. Solid-State Circuits, vol. 30, no. 3, pp. 306-310, 1995.

[2] T. J. Harpster, B. Stark, and K. Najafi, "A passive wireless integrated humidity sensor," Sens. Actuators, vol. A95, pp. 100-107, 2002.

[3] Q. Huang and M. Oberle, "A $05 \mathrm{~mW}$ passive telemetry IC for biomedical applications," J. Solid-State Circuits, vol. 33, no. 7, pp. 937-946, 1998.

[4] K. W. Fernald, J. J. Paulos, B. A. Stackhouse, and R. A. Heaton, "A selftuning digital telemetry IC for use in a microprocessor-based implantable instrument," J. Solid-State Circuits, vol. 27, no. 12, pp. 1826-1832, 1992.
[5] R. Puers, G. Vandevoorde, and D. De Bruyker, "Electrodeposited copper inductors for intraocular pressure telemetry," J. Micromech. Microeng., vol. 10, pp. 124-129, 2000.

[6] T. Akin, K. Najafi, and R. M. Bradley, "A wireless implantable multichannel digital neural recording system for a micromachined sieve electrode," J. Solid-State Circuits, vol. 33, no. 1, pp. 109-118, 1998.

[7] W. Liu, K. Vichienchom, M. Clements, S. C. DeMarco, C. Hughes, E. McGucken, M. S. Humayun, E. de Juan, J. D. Weiland, and R. Greenberg, "A neuro-stimulus chip with telemetry unit for retinal prothestic device," J. Solid-State Circuits, vol. 35, no. 10, pp. 1487-1497, 2000.

[8] M. Takeda, "Application of MEMS to industrial inspection," in Proc. 14th IEEE Int. Conf. Micro Electro Mech. Syst. Jan. 21-25, 2001, pp. 182191.

[9] T. Yasuda, I. Shimoyama, and H. Miura, "Microrobot actuated by a vibration energy field," Sens. Actuators A, Phys., vol. 43, pp. 366-370, 1994.

[10] T. Ebefors, J. U. Mattsson, E. Kälvesten, and G. Stemme, "A walking silicon microrobot," in Proc. Transducers' 99, pp. 1202-1205.

[11] P. E. Kladitis, V. M. Bright, K. F. Harsh, and Y. C. Lee, "Prototype microrobots for micro positioning in a manufacturing process and micro unmanned vehicles," in Proc. 12th IEEE Int. Conf. Micro Electro Mech. Syst. Jan. 17-21, 1999, pp. 570-575.

[12] M. Mita, M. Arai, S. Tensaka, D. Kobayashi, P. Basset, A. Kaiser, P. Masquelier, L. Buchaillot, D. Collard, and H. Fujita, "Electrostatic impact-drive microactuator," in Proc. 14th IEEE Int. Conf. Micro Electro Mech. Syst. Jan. 21-25, 2001, pp. 590-593.

[13] S. Hollar, A. Flynn, C. Bellew, and K. S. J. Pister, "Solar powered $10 \mathrm{mg}$ silicon robot," in Proc. 16th IEEE Int. Conf. Micro Electro Mech. Syst. Jan. 19-23, 2003, pp. 706-711.

[14] M. Ataka, A. Omodaka, N. Takeshima, and H. Fujita, "Fabrication and operation of polyimide bimorph actuators for a ciliary motion system," $J$. Microelectromech. Syst., vol. 2, no. 4, pp. 146-150, 1993.

[15] T. Ebefors, J. U. Mattsson, and E. Kälvesten, "A walking silicon microrobot," J. Micromech. Microeng., vol. 10, pp. 337-349, 2000.

[16] G. Vandevoorde and R. Puers, "Wireless energy transfer for stand-alone systems: A comparison between low and high power applicability," Sens. Actuators A, Phys., vol. 92, pp. 305-311, 2001.

[17] S. Takeuchi and I. Shimoyama, "Selective drive of electrostatic actuators using remote inductive powering," Sens. Actuators, vol. A95, pp. 269273, 2002.

[18] K. Minami, S. Kawamura, and M. Esashi, "Fabrication of distributed electrostatic micro actuator (DEMA)," J. Microelectromech. Syst., vol. 2, no. 3, pp. 121-127, 1993 .

[19] H. Fujita, "Future of actuators and microsystems," Sens. Actuators, vol. A56, pp. 105-106, 1996.

[20] S. Konishi and H. Fujita, "A conveyance system using air flow based on the concept of distributed micro motion systems," J. Microelectromech. Syst., vol. 3, no. 2, pp. 54-58, 1994.

[21] I. Kohelberger, H. Fujita, and R. Zurawski, "Evolutionary optimization of distributed part handling control," in Proc. IEEE Int. Conf. Intell. process. Syst., 1997, pp. 558-562.

[22] Y. Fukuta, Y.-A. Chapuis, Y. Mita, and H. Fujita, "A MEMS array for pneumatic conveyor and its control based on distributed system," in Proc. 18th IEEE Int. Conf. Micro Electro Mech. Syst., Jan. 30-Feb. 3, 2005, pp. $40-43$.

[23] M. Edo, Y. Watanabe, O. Morita, H. Nakazawa, and E. Yonezawa, "Two dimensional micro-conveyer with integrated electrostatic actuators," in Proc. 12th IEEE Int. Conf. Micro Electro Mech. Syst., Jan. 17-21, 1999, pp. 43-48.

[24] Y. Mita, D. Collard, M. Mita, and H. Fujita, "Fabrication of an inverted scratch drive actuator for a powerful and precise conveyance system," Trans. Inst. Electr. Eng. Jpn., vol. 119E, pp. 1-2, 1999.

[25] H. Nakazawa, Y. Watanabe, O. Morita, M. Edo, and E. Yonezawa, "The two-dimensional micro conveyer," in Proc. Transducers' 97, pp. 33-36.

[26] P. Basset, A. Kaiser, P. Bigotte, D. Collard, and L. Buchaillot, "A large stepwise motion electrostatic actuator for a wireless microrobot," in Proc. 15th IEEE Int. Conf. Micro Electro Mech. Syst. Jan. 20-24, 2002, pp. 606609.

[27] E. Quevy, L. Buchaillot, and D. Collard, "3-D self-assembling and actuation of electrostatic micro-structures," IEEE Trans. Electron Devices, vol. 48, no. 8, pp. 1833-1839, Aug. 2001.

[28] T. H. Lee, The Design of CMOS Radio Frequency Integrated Circuit. New York: Cambridge Univ. Press, 1998, p. 98.

[29] K. Finkenzeller, RFID Handbook. New York: Wiley, 1999, p. 68. 
[30] J. Craninckx and M. S. J. Steyaert, "A 1.8-GHz low phase noise CMOS VCO using optimized hollow spiral inductors," IEEE J. Solid-State Circuits, vol. 32, no. 5, pp. 736-744, May 1997.

[31] P. Basset, A. Kaiser, D. Collard, and L. Buchaillot, "Process and realization of a 3-D gold electroplated antenna on a flexible epoxy film for wireless micro-motion system," J. Vac. Sci. Technol., vol. B20, no. 4, pp. 14651470, Jul./Aug. 2002.

[32] B. Stefanelli, Y. Mita, A. Kaiser, and H. Fujita, "A 32 bit 100 V switching array IC ready-to-use for everyone through multi-chip foundry service," in Proc. Transducer'99, pp. 822-823.

[33] T. Akiyama, D. Collard, and H. Fujita, "Scratch drive actuator with mechanical links for self-assembly of three-dimensional MEMS," J. Microelectromech. Syst., vol. 6, pp. 10-17, 1997.

[34] C. P. Yue and S. S. Wong, "On-chip spiral inductors with patterned ground shields for Si-based RF IC's," J. Solid-State Circuits, vol. 33, no. 5, pp. 743-747, May 1998.

[35] J. M. Kahn, R. H. Katz, and K. S. J. Pister, "Next century challenges: Mobile networking for smart dust," in Proc. ACM/IEEE Int. Conf. Mobile Comput. Netw., Seattle, WA, Aug. 17-19, 1999, pp. 271-287.

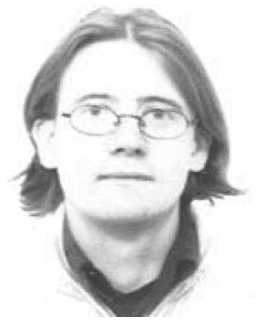

Philippe Basset was born in 1972. He received the B.S. degree in electrical engineering from the University of Orsay, Orsay, France, in 1994, and the M.S. and $\mathrm{Ph} . \mathrm{D}$. degrees in electrical engineering from the University of Lille, Lille, France, in 1997 and 2003, respectively.

In 2004, he was a Postdoctoral Fellow at the Carnegie Mellon University, Pittsburgh, PA. In 2005, he joined the Ecole Supérieure d'Electronique et Electrotechnique (ESIEE), Noisy-le-Grand, France, where he is currently an Assistant Professor. His research interests include MEMS sensors and actuators, and micropower sources for autonomous MEMS.

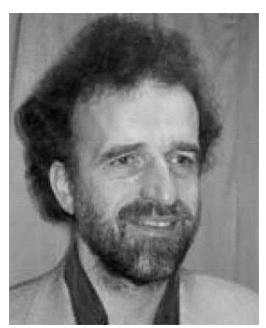

Andreas Kaiser ( $\left.\mathrm{S}^{\prime} 85-\mathrm{M}^{\prime} 87\right)$ received the engineering diploma from the Institut Supérieur d'Electronique du Nord (ISEN), Lille, France, in 1984, and the Ph.D. and HDR degrees from the University of Lille, Lille, in 1990 and 1998, respectively.

In 1990, he joined the Centre National de la Recherche Scientifique (CNRS) where he is a leader in the Analog/RF IC Design Group at the Institut d'Electronique, de Microelectronique et de Nanotechnologies (IEMN), Villeneuve-d'Ascq, France. Currently, he is a Professor at ISEN. He is the author or coauthor of several published papers on continuous and discrete time analog circuits, data-converters, RF-MEMS, and analogue design automations.

Dr. Kaiser was the Programme Chairman of the European Solid State Circuits Conference in 1995 and 2005.

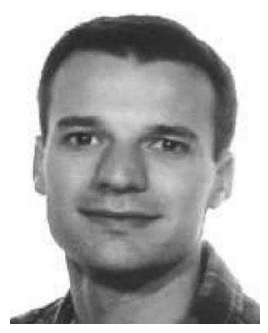

Bernard Legrand received the B.S., M.S., and the $\mathrm{Ph} . \mathrm{D}$. degrees in electronics from the Université des Sciences et Technologies de Lille, Villeneuve d'Ascq, France, in 1991, 1996, and 2000, respectively.

In 2000, he joined the Silicon Microsystem Group, Institut d'Electronique, de Microelectronique et de Nanotechnologies (IEMN), Lille, France. His research was first focussed on MEMS resonators and their electrical characterization. His current research interests include micromechanical sensors and electrostatic actuators with ultimate performances for the characterization of nano-objects and for operation in a liquid environment, mechanical interactions between biological cells, and effects of mechanical stress on their response. Since 2001, he has been a Research Scientist with the Centre National de la Recherche Scientifique (CNRS), Villeneuve d'Ascq, France.

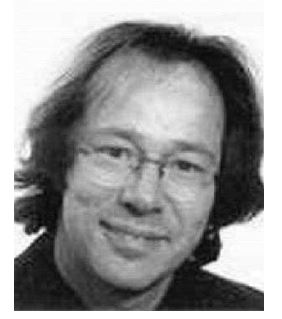

Dominique Collard (M'01) was born in Cambrai, France, in 1958. He received the Engineering degree from the Institut Supérieur d'Electronique et du Numérique (ISEN), Lille, France, in 1980, and the $\mathrm{Ph} . \mathrm{D}$. degree from the University of Lille, Lille, in 1984. His study focussed on silicon processes and devices simulations.

From 1985 to 1986 , he was a Visiting Scientist at the Toshiba Ultra Large Scale Integration (ULSI) Research Center, Kawasaki, Japan. In 1988, he joined the Centre National de la Recherche Scientifique (CNRS), as a Senior Researcher, and established a research group on process and device simulation at ISEN and Institut d'Electronique, de Microélectronique et de Nanotechnologie (IEMN), Lille, France. His research activity was focused on silicon oxidation simulation and related stress effects. From 1995 to 1997, he was a Director of the Laboratory for Integrated Micromechatronic Systems (LIMMS), Tokyo, Japan, a joint CNRS laboratory with the Institute of Industrial Science and the University of Tokyo, Tokyo. At LIMMS, his work involved silicon-based electrostatic actuators for device alignment systems and selfassembling three-dimensional (3-D) technologies. In 1997, he joined IEMN, as the CNRS Research Director and established the Silicon Micro-System Group, and worked on microactuators and their integration in silicon microtechnology. Since November 2000, he has been a Professor at the University of Tokyo, and a Director of the Center of International Research on Micromechatronics (CIRMM)/CNRS, a joint center between the Institute of Industrial Science (IIS), the University of Tokyo, and the CNRS to promote joint research projects between French and Japanese laboratories in micro and nano systems. In August 2005, he again joined LIMMS. His current research interests include siliconbased microsystems, the integration of microtechnologies for communication systems.

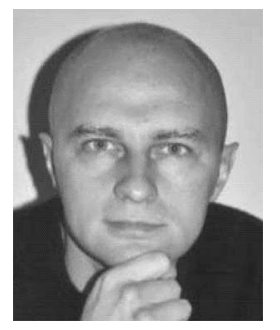

Lionel Buchaillot received the Diplôme d'Etudes Approfondies (DEA) degree in material sciences and the Ph.D. degree in mechanical engineering from the Université de Franche-Comté, Besançon, France, in 1991 and 1995 , respectively.

From 1995 to 1997, he was with the Laboratory for Integrated Micromechatronic Systems (LIMMS)/Centre National de la Recherche Scientifique (CNRS)-Institute of Industrial Science (IIS), Tokyo, Japan, as a Japan Society for Promotion of Science (JSPS) Fellow, engaged in thin-film shape memory alloys actuators for MEMS. In 1997, he was a Research and Development Engineer with SFIM (now SAGEM) and AVIAC Technologies. In 1998 he joined the Institut Supérieur d'Electronique et du Numérique (ISEN), Institut d'Electronique, de Microelectronique et de Nanotechnologie (IEMN), CNRS, Villeneuve d'Ascq, France, where he is currently the Head of the Silicon-based MEMS Research Group. His current research interests include RF MEMS, MEMS reliability, and MEMS for nanotechnology. 